BMJ Surgery, Interventions, $\&$ Health Technologies

\section{Catheter-related complications and mortality of atrial fibrillation ablation following introduction of contact force- sensing technology}

To cite: Akar JG, Hummel JP, Yao X, et al. Catheter-related complications and mortality of atrial fibrillation ablation following introduction of contact force-sensing technology. BMJ Surg Interv Health Technologies 2020;2:e000058. doi:10.1136/ bmjsit-2020-000058

- Additional material is published online only. To view, please visit the journal online (http://dx.doi.org/10.1136/ bmjsit-2020-000058)

Received 21 July 2020 Revised 10 November 2020 Accepted 14 December 2020

Check for updates

(c) Author(s) (or their employer(s)) 2020. Re-use permitted under CC BY-NC. No commercial re-use. See rights and permissions. Published by BMJ.

For numbered affiliations see end of article.

Correspondence to Dr Peter A Noseworthy; noseworthy.peter@mayo.edu

\section{ABSTRACT}

Objectives Contact force-sensing catheters allow realtime catheter-tissue contact force monitoring during atrial fibrillation. These catheters were rapidly adopted into clinical practice following market introduction in 2014, but concerns have been raised regarding collateral damage such as esophageal injury. We sought to examine whether the introduction of force-sensing catheters was associated with a change in short-term and intermediate-term acute care use, complications and mortality following atrial fibrillation ablation.

Design Retrospective cohort analysis. We used inverse probability treatment weight matching to account for the differences in baseline characteristics between groups.

Setting We examined patients included in the OptumLabs Data Warehouse who underwent ablation for atrial fibrillation before (2011-2013) and after (2015-2017) the market introduction of contact force-sensing catheters. Main outcome measures We examined 30-day and 90-day rates of all-cause acute care use, including hospitalizations and emergency department visits, as well as death and hospitalization for catheter-related complications, including atrioesophageal fistula, pericarditis, cardiac tamponade/perforation and stroke/ transient ischemic attack.

Results Our sample included 3470 and 5772 patients who underwent atrial fibrillation (AF) ablation before and after market introduction of contact force-sensing catheters, respectively. Complication rates were low and did not differ between the two periods ( $p>0.10$ for each outcome). The 30-day and 90-day mortality was $0.1 \%$ and $0.3 \%$, respectively after market introduction and unchanged from prior to 2014. The 90-day rates of all-cause acute care use decreased, from $27.0 \%$ in $2011-$ 2013 to $23.9 \%$ in $2015-2017$ ( $p<0.001)$.

Conclusions AF ablation-related catheter complications and mortality are low and there has been no significant change following the introduction of force-sensing catheters.

\section{INTRODUCTION}

The incidence of short-term and intermediate-term safety outcomes following catheter ablation of atrial fibrillation (AF)

\section{Key messages}

What is already known about this subject?

- Previous studies examining complications following atrial fibrillation (AF) ablation are limited in their application to contemporary practice.

- Force-sensing catheter technology was rapidly and widely adopted for AF ablation procedures, however there are limited data on complications related to the use of these catheters.

- Force-sensing catheters produce more extensive ablation lesions that could predispose to complications related to cardiac perforation and collateral damage to the esophagus.

What are the new findings?

- We performed a retrospective cohort analysis using OptumLabs Data Warehouse to compare rates of adverse outcomes among patients undergoing ablation before $(n=3470)$ and after $(n=5772)$ the market introduction of contact force-sensing catheters.

- Complication rates were low and did not differ between the two periods ( $p>0.10$ for each outcome).

- The 30-day and 90 -day mortality was $0.1 \%$ and $0.3 \%$, respectively and did not change after introduction of force sensing technology.

- The 90-day rates of all-cause acute care use decreased from $27.0 \%$ to $23.9 \%$ after introduction of force sensing technology $(p<0.001)$.

\section{How might these results affect future} research or surgical practice?

- The introduction of contact-force catheter technology was not associated with increasing rates of 30 day or 90-day mortality, or serious peri-procedural complications such as atrial perforation.

- The real-world use of these catheters does not appear to be associated with increased rates of hospitalization or emergency visits.

requires continual reappraisal. Not only has the AF ablation technique evolved from segmental pulmonary vein ablation to wide area circumferential isolation with possible 
substrate modification, but there have also been significant advances in mapping and ablation catheter technology to improve procedural safety and effectiveness. Previous studies examining complications following AF ablation are limited in their application to contemporary practice because they preceded the introduction of currently used catheters capable of force-sensing and increased power delivery to the posterior left atrial wall. ${ }^{1-5}$ Furthermore, there are limited data on specific procedure-related complications that occur in the shortterm and intermediate-term such as pericarditis and atrioesophageal fistula (AEF) in a contemporary cohort. ${ }^{6}$

The most significant recent advance in catheter technology has been the ability to measure contact force. Contact force-sensing catheters were introduced to the US market in early 2014 and have been rapidly and widely adopted. Contact force-sensing catheters are not dependent on tactile sensation and allow titration of contact force resulting in more consistent transmural ablation lesions. ${ }^{7-9}$ While this is generally desirable for procedural success, the deeper ablation lesions could predispose to procedural complications, especially those related to cardiac perforation and collateral damage to adjacent structures. A recent analysis of the Food and Drug Administration's Manufacturer and User Facility Device Experience database suggested that use of contact force-sensing catheters may be associated with increased risk for AEF formation. ${ }^{10}$

To evaluate these concerns and provide contemporary safety data, we examined real-world outcomes following AF ablation using data from a large, national contemporary population of commercially insured patients and Medicare Advantage beneficiaries. Specifically, we examined whether the introduction of force-sensing catheters was associated with an increase in short-term and intermediate-term acute care use (hospitalization and emergency department (ED) visits), death, and complications, particularly those related to cardiac perforation including AEF.

\section{METHODS}

\section{Data source}

We conducted a retrospective cohort analysis using OptumLabs Data Warehouse, a large US database with de-identified administrative claims data for individuals enrolled in private and Medicare Advantage health plans. ${ }^{11}$ All ages, ethnicities, and racial groups are represented in the database spanning all 50 states. Medical claims include information on physician, hospital, and outpatient prescription services. ${ }^{12}$

\section{Study population}

The study population included adult patients ( $\geq 18$ years) with AF who underwent AF ablation between January 1, 2011 and September 30, 2017. The patients were identified using International Classification of Diseases (ICD)-9 and ICD-10 diagnostic codes for AF, combined with Current Procedural Terminology (CPT) procedural codes for $\mathrm{AF}$ ablation. If a patient received multiple ablations, the date of the first ablation was defined as the index date. All patients were required to have continuous medical enrollment for at least 12 months prior to the index procedure and 90 days post the index procedure or death. The Mayo Clinic Institutional Review Board exempted this study from review because the study used pre-existing, deidentified data.

Because contact force-sensing technology was introduced in 2014, we excluded patients who underwent AF ablation during this time. Essentially, we used this as a wash-out period, recognizing that while there was widespread adoption, time was needed for cardiac electrophysiology labs to use already purchased catheters and replace existing stock, exchanging contact force-sensing catheters for traditional catheters.

\section{Covariates}

Independent variables of interest at baseline were demographics: age, gender, and race (white, black, Hispanic, Asian, or unknown), region (Midwest, Northeast, South, or West) and baseline clinical characteristics: anemia, vascular disease, chronic obstructive pulmonary disease, obesity, hypertension, diabetes, renal disease, congestive heart failure, history of cardioversion, or stroke. These factors included all components of the HAS-BLED (a bleeding risk score) and $\mathrm{CHA}_{2} \mathrm{DS}_{2}$-VASc (a stroke risk score) scores. We also included baseline prescriptions for amiodarone. Comorbidities were captured by ICD-9 and ICD-10 codes in any position on claims in the 12 months prior to the index ablation procedure. Use of amiodarone in the 90 days prior to index ablation date was determined based on pharmacy claims.

\section{Primary and secondary outcomes}

Our primary outcome was all-cause acute care use, including the immediate peri-procedural period (index hospitalization for ablation), as well as subsequent ED visits and hospitalizations, within 30 days and 90 days of AF ablation. Secondary outcomes were all-cause mortality and ablation-specific complications within 30 days and 90 days of ablation. Ablation-specific complications encountered during the index hospitalization included cardiac perforation resulting in tamponade or need for urgent intervention, pericarditis, stroke or transient ischemic attack (TIA), and AEF (see online supplemental appendix table 1 for pertinent ICD-9, ICD-10, and CPT codes). Catheter-related complications were identified using primary and secondary discharge diagnosis codes (see online supplemental appendix table 2 for pertinent ICD-9, ICD-10, and CPT codes). The codes and algorithms used herein have been commonly used and validated in many previous studies. ${ }^{13} 14$

Mortality was identified based on the Social Security Death Master File and patient discharge status.

Since AEF is difficult to assess with a single diagnosis code, we used a hierarchical method incorporating 
multiple codes as outlined in online supplemental appendix table 3. In brief, AEF was assessed as either definite or probable based on diagnosis codes occurring within 90 days of ablation. Definite AEF was defined as: (1) a diagnosis code for AEF or esophageal injury associated with death; or (2) an esophageal intervention associated with a diagnosis of either AEF, esophageal injury, mediastinal infection, air embolism, or hematemesis. Probable AEF was defined when: (1) patients underwent an esophageal intervention and had other possible signs of AEF including stroke, multi-organ failure, infection/sepsis, altered mental status, fever chest pain, or dysphagia; or (2) codes for mediastinal infection, air embolism, or hematemesis were associated with death; or (3) codes for both stroke and infection/sepsis were associated with death.

In order to reflect the known clinical difficulty in establishing a diagnosis of AEF, we performed a sensitivity analysis in which AEF was defined as any two of the diagnosis codes occurring within 90 days of ablation: mediastinal infection, air embolism, hematemesis, stroke/TIA, multiorgan failure, infection/sepsis, altered mental status, fever, chest pain, or dysphagia.

\section{Statistical analyses}

To examine the association of the contact force-sensing technology introduced in 2014 on the risk of several outcomes, we created a balanced cohort (before 20112013 vs after 2015-2017). We used inverse probability of treatment weighting (IPTW) to balance covariates between the two time periods. The underlying propensity model included the demographics, comorbidities, and baseline medication use shown in table 1 . We evaluated the balance among the two time periods by comparing standardized mean differences of baseline covariates. A baseline characteristic was considered balanced if the standardized mean difference was $<10 \%$. We used a logistic regression to compare treatments in the weighted population. All analyses were conducted using SAS software V.9.4 and Stata V.15.1.

Table 1 Patient characteristics before and after IPTW.

\begin{tabular}{|c|c|c|c|c|c|c|}
\hline & \multicolumn{3}{|c|}{ Before IPTW } & \multicolumn{3}{|l|}{ After IPTW } \\
\hline & $\begin{array}{l}2011-2013 \\
(N=3470)\end{array}$ & $\begin{array}{l}2015-2017 \\
(N=5772)\end{array}$ & Std. Diff. (\%) & $\begin{array}{l}2011-2013 \\
(N=3470)\end{array}$ & $\begin{array}{l}2015-2017 \\
(N=5772)\end{array}$ & Std. Diff. (\%) \\
\hline \multicolumn{7}{|l|}{ Gender } \\
\hline Female & $28.60 \%$ & $33.90 \%$ & 11.30 & $31.60 \%$ & $31.80 \%$ & 0.50 \\
\hline \multicolumn{7}{|l|}{ Race } \\
\hline White & $82.20 \%$ & $82.30 \%$ & 4.00 & $82.40 \%$ & $82.30 \%$ & 0.20 \\
\hline Black & $8.80 \%$ & $7.70 \%$ & 4.30 & $8.10 \%$ & $8.10 \%$ & 0.00 \\
\hline Hispanic & $4.30 \%$ & $5.10 \%$ & 3.70 & $4.80 \%$ & $4.80 \%$ & 0.40 \\
\hline Asian & $1.70 \%$ & $1.60 \%$ & 1.00 & $1.60 \%$ & $1.60 \%$ & 0.10 \\
\hline Anemia & $21.00 \%$ & $19.00 \%$ & 4.40 & $20.00 \%$ & $20.00 \%$ & 0.20 \\
\hline Vascular disease & $24.00 \%$ & $27.00 \%$ & 5.90 & $26.00 \%$ & $26.00 \%$ & 0.20 \\
\hline COPD & $6.00 \%$ & $5.00 \%$ & 3.00 & $6.00 \%$ & $6.00 \%$ & 0.10 \\
\hline Obesity & $31.00 \%$ & $43.00 \%$ & 23.80 & $38.00 \%$ & $38.00 \%$ & 0.30 \\
\hline Hypertension & $78.00 \%$ & $81.00 \%$ & 7.40 & $80.00 \%$ & $80.00 \%$ & 0.30 \\
\hline Diabetes & $19.00 \%$ & $23.00 \%$ & 10.40 & $22.00 \%$ & $21.00 \%$ & 0.90 \\
\hline Renal disease & $7.00 \%$ & $12.00 \%$ & 16.20 & $10.00 \%$ & $10.00 \%$ & 0.80 \\
\hline $\mathrm{CHF}$ & $24.00 \%$ & $28.00 \%$ & 8.60 & $27.00 \%$ & $27.00 \%$ & 0.90 \\
\hline
\end{tabular}

*Not included in IPTW.

CHF, congestive heart failure; COPD, chronic obstructive pulmonary disease; IPTW, inverse probability of treatment weighting. 
Table 2 Clinical events before (2011-2013) and after (20152017) introduction of force-sensing catheters.

\begin{tabular}{|c|c|c|c|}
\hline & $\begin{array}{l}\text { Before } \\
(2011-2013) \\
n=3470(\%)\end{array}$ & $\begin{array}{l}\text { After } \\
\text { (2015-2017) } \\
n=5772(\%)\end{array}$ & $P$ value \\
\hline \multicolumn{4}{|l|}{ 30-day events } \\
\hline AEF & 0.0 & 0.01 & - \\
\hline Definite & 0.0 & 0.01 & - \\
\hline Probable & 0.0 & 0.01 & - \\
\hline Sensitivity & 1.1 & 0.9 & 0.247 \\
\hline Death & 0.2 & 0.1 & 0.309 \\
\hline Tamponade or intervention & 0.2 & 0.3 & 0.321 \\
\hline Pericarditis & 1.4 & 1.5 & 0.735 \\
\hline Stroke/TIA & 0.1 & 0.1 & 0.630 \\
\hline Hospitalization or ED visit & 18.1 & 16.8 & 0.134 \\
\hline \multicolumn{4}{|l|}{ 90-day events } \\
\hline AEF & 0.04 & 0.09 & 0.379 \\
\hline Definite & 0.04 & 0.04 & 0.942 \\
\hline Probable & 0.04 & 0.07 & 0.580 \\
\hline Sensitivity & 2.2 & 1.6 & 0.039 \\
\hline Death & 0.5 & 0.3 & 0.226 \\
\hline Tamponade or intervention & 0.4 & 0.5 & 0.494 \\
\hline Pericarditis & 1.7 & 1.8 & 0.675 \\
\hline Stroke/TIA & 0.2 & 0.2 & 0.960 \\
\hline Hospitalization or ED visit & 27.0 & 23.9 & 0.001 \\
\hline
\end{tabular}

$\mathrm{AEF}$, atrioesophageal fistula; $\mathrm{ED}$, emergency department; TIA, transient ischemic attack.

\section{RESULTS}

\section{Patient characteristics}

Table 1 shows the baseline characteristics of patients undergoing AF ablation before and after introduction of contact force-sensing catheters, including 3470 patients who underwent ablation between 2011 and 2013 and 5722 patients who underwent ablation between 2015 and 2017. Patients undergoing ablation after 2014 were older, more obese, more likely females and with lower history of stroke. As demonstrated in the right panel of table 1, the groups were well-balanced following inverse probability weight matching, with all standardized mean differences $<1 \%$. There was no significant difference in age, gender, race, or comorbidities. The majority of patients included in our sample were men and white, and most had a history of hypertension. The mean $\mathrm{CHA}_{2} \mathrm{DS}_{2}$-VASc score was 2.6 in both groups between 2011-2013 and 2015-2017, respectively. The mean HAS-BLED score was 1.8 and 1.9 , respectively.

\section{Outcomes}

The overall adjusted rates of short-term and intermediateterm all-cause emergency visits and hospitalizations are reported in table 2 . There was a statistically significant reduction in the 90-day rates of all-cause acute care use, including ED visits and hospitalizations, in the years following market introduction of the contact-sensing catheters, from $27.0 \%$ to $23.9 \%$ ( $\mathrm{p}<0.001)$. Prior to the introduction of contact force-sensing catheters, $18.1 \%$ of patients had at least one hospitalization or emergency visit within 30 days of undergoing $\mathrm{AF}$ ablation, compared with $16.8 \%$ in the years following the introduction of contact force-sensing catheters $(\mathrm{p}=0.134)$.

Adjusted rates of short-term and intermediate-term risk of mortality and procedural complications are also reported in table 2. We found no difference in the secondary outcomes of mortality and procedural complications before and after the market introduction of contact force-sensing catheters. The 30-day and 90-day mortality rates were not significantly changed after market introduction of force-sensing catheters $(0.2 \%$ and $0.5 \%$ before vs $0.1 \%$ and $0.3 \%$ after, respectively; $p$ values $>0.10)$. Specifically, there was no difference in 30-day and 90-day risk of AEF $(0.0 \%$ and $0.04 \%$ before vs $0.01 \%$ and $0.09 \%$ after; $p>0.10$ ), tamponade or intervention $(0.2 \%$ and $0.4 \%$ before vs $0.3 \%$ and $0.5 \%$ after; $\mathrm{p}>0.3)$ and stroke $(0.1 \%$ and $0.2 \%$ before vs $0.1 \%$ and $0.2 \%$ after; $\mathrm{p}>0.6$ ).

\section{DISCUSSION}

We examined short-term and intermediate-term safety outcomes of patients undergoing AF ablation following the introduction and widespread adoption of contactforce sensing catheters in 2014, compared with outcomes observed among patients for whom previously available standard catheters were used. The main findings of analysis include: (1) the introduction of contact-force catheter technology did not appear to be associated with increasing rates of death and serious peri-procedural complications, including short-term and intermediateterm adverse events; (2) specifically, the introduction of contact-force catheter technology did not appear to be associated with increased risk of AEF or cardiac perforation; (3) the introduction of contact-force catheter technology did not appear to be associated with increased rates of hospitalization or emergency visits and may have been associated with a reduction in overall healthcare utilization at 90 days.

This study compares real-world, modern-era outcomes in a large, national adjusted cohort. Contrary to previous unadjusted reports, we found that AF ablation as practiced in the modern era following introduction of forcesensing catheters does not carry an increased risk of AEF or cardiac perforation. Our study suggests that the use of contact force-sensing catheters carries a small, approximately $0.1 \%$, risk of AEF as well as low rate of mortality and morbidity due to serious adverse events related to stroke, cardiac perforation, hospitalizations, and emergency room visits. While this study did not examine the effectiveness of $\mathrm{AF}$ ablation following the introduction of force-sensing catheters, the low rates of adverse outcomes of $\mathrm{AF}$ ablation as practiced in the real world is highly encouraging and consistent with recent reports examining short-term events. ${ }^{6}$ A recent analysis of AF ablation 
trends using the Nationwide Readmissions Database from 2010 to 2015 reported a 30-day inpatient mortality rate of $0.46 \%$, which is higher than our study despite not capturing out-of-hospital deaths, readmissions across different states, or long-term complications that can occur after 30 days (eg, AEF). It is worth noting that the Nationwide Readmissions Database contains only AF ablations performed among hospitalized patients, which likely represents a higher risk patient population given that the majority of contemporary AF ablation procedures are performed in the ambulatory setting. Another recent analysis of demonstrated that both contact force and non-contact force ablation catheters have similarly low incidence of AEF, however this analysis was derived only from ablation device manufacturer's complaint database. ${ }^{15}$ Thus, in comparison to other studies, our analysis has the advantage of being both contemporaneous and representative of broad populations of patients undergoing $\mathrm{AF}$ ablation in routine practice.

AEF is one of the most morbid and lethal complications of catheter ablation of AF. While initially thought to be exceedingly rare, the incidence of AEF is now being revised and is thought to be higher than originally expected, with current estimates ranging from $0.02 \%$ to $0.11 \% .{ }^{16-20}$ Given the increase in $\mathrm{AF}$ ablation procedures in recent years, AEF continues to be among the most feared complications. However, the actual incidence of AEF has likely been underestimated due to the difficulty in establishing the diagnosis and its association with the $\mathrm{AF}$ ablation procedure. Patients with AEF usually present 2-4 weeks following the index ablation procedure with variable symptoms of fever, sepsis, hematemesis, or stroke. ${ }^{16}$ Given the acuity and non-specificity of the symptoms, the patient may not be referred back to the institution where the index ablation is performed, and an association with the AF ablation procedure may be overlooked. Thus, the diagnosis of AEF may be missed and/or under-reported in single-center studies. Furthermore, given the temporal delay in the formation and clinical presentation of AEF, studies that examine acute or short-term peri-procedural complications are likely to miss or under-report this complication. Our study examined multiple algorithms as sensitivity analyses and did not find an increase in AEF over time.

Previous reports examining the period between 2000 and 2010 have demonstrated similarly low rates of serious complications with AF ablation, but these studies generally examined acute peri-procedural complications and did not examine potential adverse outcomes that may occur in the intermediate term following $\mathrm{AF}$ ablation, such as $\mathrm{AEF}$ or late-presenting tamponade. These studies also preceded the use of contact force-sensing technology and thus are not representative of modern practice. More recent studies examining $\mathrm{AF}$ ablation from single tertiary referral centers probably underestimated the true incidence of procedurerelated death, AEF and other major complications, both because these centers tend to be more specialized and perform a larger number of procedures, but also because the centers tend to have greater experience, having used the novel technologies for a longer period of time. Our study is the first claims-based examination of $\mathrm{AF}$ ablation in the modern era to focus on intermediate-term complications and mortality, thereby capturing all hospitalizations regardless of institution or geography.

\section{Limitations}

There are several limitations that should be considered. First, using claims-based data, we are unable to identify the exact catheters used for ablation, as this information is not available. Therefore, our finding of no increasing rates of mortality and complications following the introduction of contact-force ablation catheters does not lead to a firm conclusion of no increased risks associated with this technology because it might have been confounded by other factors such as the increasing adoption of cryoballoon catheters in recent years. Given the rapid adoption of contact force-sensing catheters, it is reasonable to assume that these catheters were increasingly used after the 12-month blanking period following their introduction in 2014. Nevertheless, this limitation emphasizes the importance of integrating the Unique Device Identifier into claims, ${ }^{22}$ which would enable future studies to explicitly compare outcomes among patients for whom different catheters are used. Second, while we found no trends of increased risk of safety outcomes after the introduction of contact-force catheters, we cannot rule out residual confounding despite propensity risk adjustment. In addition, we emphasize that the ascertainment of outcomes and covariates in this study relied on administrative data/claims which are subject to misclassification. No manual chart review to validate individual codes was feasible given the de-identified nature of the database. However, there would be no systematic ascertainment differences between the two treatment groups, and any potential misclassification should be non-differential and should not influence estimated treatment effects. In addition, the diagnosis and procedure codes used in this study have demonstrated good performance in validation studies with positive predictive values around $90 \% .^{1423-29}$

\section{CONCLUSIONS}

The introduction of contact-force catheter technology in the modern era was not associated with increasing rates of 30-day or 90-day mortality, or serious peri-procedural complications, including short-term and intermediate-term serious adverse events and, importantly, AEFs. Furthermore, real-world use of these catheters does not appear to be associated with increased rates of hospitalization or emergency visits. Continued efforts are needed to monitor contemporary use of novel technologies to ensure that patients are achieving higher-quality care outcomes.

\section{Author affiliations}

${ }^{1}$ Department of Internal Medicine, Cardiovascular Medicine, Yale University School of Medicine, New Haven, Connecticut, USA

${ }^{2}$ Department of Medicine, University of Wisconsin-Madison School of Medicine and Public Health, Madison, Wisconsin, USA 
${ }^{3}$ Division of Health Care Policy and Research, Mayo Clinic, Rochester, Minnesota, USA

${ }^{4}$ Robert D. and Patricia E. Kern Center for the Science of Health Care Delivery, Mayo Clinic, Rochester, Minnesota, USA

${ }^{5}$ University of California San Francisco School of Medicine, San Francisco, California, USA

${ }^{6}$ Center for Devices and Radiological Health, US Food and Drug Administration, Silver Spring, Maryland, USA

${ }^{7}$ Internal Medicine, Yale University School of Medicine, New Haven, Connecticut, USA

Contributors Concept and design: JGA, JPH, JR, PN, NDS. Acquisition, analysis, or interpretation of data: JGA, JPH, XY, LS, SD, JD, RW, NDS, JR, PN. Drafting of the manuscript: JGA, PN, SD. Critical revision of the manuscript for important intellectual content: JGA, JPH, XY, LS, SD, JD, RW, NDS, JR, PN. Statistical analysis: LS, XY. Obtained funding: JR, NDS. Supervision: $n / a$.

Funding This study was supported by the Food and Drug Administration (FDA) of the US Department of Health and Human Services (HHS) as part of a financial assistance award by a Center of Excellence in Regulatory Science and Innovation (CERSI) grant to Yale University and Mayo Clinic from the US Food and Drug Administration (U01FD005938) totaling US\$48 000 with $100 \%$ funded by FDA/HHS

Disclaimer The contents are those of the authors and do not necessarily represent the official views of the HHS or FDA. Role of the Funder/Sponsor: Some of the authors (JD and RW) are employees of the FDA; however, other officials at the FDA had no role in the design and conduct of the study; the collection, analysis, and interpretation of the data; the preparation of the manuscript; or the decision to submit the manuscript for publication. The manuscript was subject to administrative review prior to submission, but the content was not altered by this review.

Competing interests SD currently receives research support through the National Institute of Health (K12HL138046) and the Greenwall Foundation. JR currently receives research support through Yale University from Johnson and Johnson to develop methods of clinical trial data sharing, from the Medical Device Innovation Consortium as part of the National Evaluation System for Health Technology (NEST), from the Agency for Healthcare Research and Quality (R01HS022882), from the National Heart, Lung and Blood Institute of the National Institutes of Health (NIH) (R01HS025164), and from the Laura and John Arnold Foundation to establish the Good Pharma Scorecard at Bioethics International and to establish the Collaboration for Research Integrity and Transparency (CRIT) at Yale. NDS has received research support through Mayo Clinic from the Centers of Medicare and Medicaid Innovation, from the Agency for Healthcare Research and Quality (R01HS025164; R01HS025402; R03HS025517; U19HS024075), from the National Heart, Lung and Blood Institute of the National Institutes of Health (NIH) (R56HL130496; R01HL131535), National Science Foundation, and from the Patient Centered Outcomes Research Institute (PCORI).

Patient consent for publication Not required.

Provenance and peer review Not commissioned; externally peer reviewed.

Data availability statement № data are available. Because we use administrative claims data from Optum Labs Data Warehouse we are unable to share with external investigators.

Open access This is an open access article distributed in accordance with the Creative Commons Attribution Non Commercial (CC BY-NC 4.0) license, which permits others to distribute, remix, adapt, build upon this work non-commercially, and license their derivative works on different terms, provided the original work is properly cited, appropriate credit is given, any changes made indicated, and the use is non-commercial. See: http://creativecommons.org/licenses/by-nc/4.0/.

\section{ORCID iD}

Peter A Noseworthy http://orcid.org/0000-0002-4308-0456

\section{REFERENCES}

1 Piccini JP, Sinner MF, Greiner MA, et al. Outcomes of Medicare beneficiaries undergoing catheter ablation for atrial fibrillation. Circulation 2012;126:2200-7.

2 Freeman JV, Tabada GH, Reynolds K, et al. Contemporary procedural complications, hospitalizations, and emergency visits after catheter ablation for atrial fibrillation. Am J Cardiol 2018;121:602-8.
3 Deshmukh A, Patel NJ, Pant S, et al. In-hospital complications associated with catheter ablation of atrial fibrillation in the United States between 2000 and 2010: analysis of 93801 procedures. Circulation 2013;128:2104-12.

4 Noseworthy PA, Gersh BJ, Kent DM, et al. Atrial fibrillation ablation in practice: assessing CABANA generalizability. Eur Heart $J$ 2019:40:1257-64.

5 Packer DL, Mark DB, Robb RA, et al. Effect of catheter ablation vs antiarrhythmic drug therapy on mortality, stroke, bleeding, and cardiac arrest among patients with atrial fibrillation: the CABANA randomized clinical trial. JAMA 2019;321:1261-74.

6 Friedman DJ, Pokorney SD, Khanna R, et al. Catheter ablation of atrial fibrillation with and without on-site cardiothoracic surgery. J Am Coll Cardiol 2019;73:2487-9.

7 Andrade JG, Monir G, Pollak SJ, et al. Pulmonary vein isolation using "contact force" ablation: the effect on dormant conduction and longterm freedom from recurrent atrial fibrillation--a prospective study. Heart Rhythm 2014;11:1919-24.

8 Marijon E, Fazaa S, Narayanan K, et al. Real-time contact force sensing for pulmonary vein isolation in the setting of paroxysmal atrial fibrillation: procedural and 1-year results. $J$ Cardiovasc Electrophysiol 2014;25:130-7.

9 Natale A, Reddy VY, Monir G, et al. Paroxysmal AF catheter ablation with a contact force sensing catheter: results of the prospective, multicenter SMART-AF trial. J Am Coll Cardiol 2014;64:647-56.

10 Black-Maier E, Pokorney SD, Barnett AS, et al. Risk of atrioesophageal fistula formation with contact force-sensing catheters. Heart Rhythm 2017;14:1328-33.

11 Wallace PJ, Shah ND, Dennen T, et al. Optum Labs: building a novel node in the learning health care system. Health Aff 2014;33:1187-94.

12 Food \& Drug Administration. Collection, analysis, and availability of demographic subgroup data for FDA-approved medical products, 2013.

13 Noseworthy PA, Kapa S, Haas LR, et al. Trends and predictors of readmission after catheter ablation for atrial fibrillation, 2009-2013. Am Heart J 2015;170:483-9.

14 Tirschwell DL, Longstreth WT. Validating administrative data in stroke research. Stroke 2002;33:2465-70.

15 Calkins H, Natale A, Gomez T, et al. Comparing rates of atrioesophageal fistula with contact force-sensing and non-contact force-sensing catheters: analysis of post-market safety surveillance data. J Interv Card Electrophysiol 2020;59:49-55.

16 Cummings JE, Schweikert RA, Saliba WI, et al. Brief communication: atrial-esophageal fistulas after radiofrequency ablation. Ann Intern Med 2006;144:572-4.

17 Mohanty S. Outcomes of atrio-esophageal fistula following catheter ablation of atrial fibrillation treated with surgical repair versus esophageal stenting. J Cardiovasc Electrophysiol 2014;25:E6.

18 Pappone C, Oral H, Santinelli V, et al. Atrio-esophageal fistula as a complication of percutaneous transcatheter ablation of atrial fibrillation. Circulation 2004;109:2724-6.

19 Scanavacca MI, D'ávila A, Parga J, et al. Left atrial-esophageal fistula following radiofrequency catheter ablation of atrial fibrillation. $J$ Cardiovasc Electrophysiol 2004;15:960-2.

20 Zellerhoff S, Lenze F, Schulz R, et al. Fatal course of esophageal stenting of an atrioesophageal fistula after atrial fibrillation ablation. Heart Rhythm 2011;8:624-6.

21 Mohanty S, Santangeli P, Mohanty P, et al. Outcomes of atrioesophageal fistula following catheter ablation of atrial fibrillation treated with surgical repair versus esophageal stenting. $J$ Cardiovasc Electrophysiol 2014;25:579-84.

22 Dhruva SS, Ross JS, Schulz WL, et al. Fulfilling the promise of unique device identifiers. Ann Intern Med 2018;169:183-5.

23 Kumamaru H, Judd SE, Curtis JR, et al. Validity of claims-based stroke algorithms in contemporary Medicare data: reasons for geographic and racial differences in stroke (regards) study linked with Medicare claims. Circ Cardiovasc Qual Outcomes 2014;7:611-9.

24 Kokotailo RA, Hill MD. Coding of stroke and stroke risk factors using International classification of diseases, revisions 9 and 10. Stroke 2005;36:1776-81.

25 Jensen PN, Johnson K, Floyd J. Identifying atrial fibrillation from electronic medical data: a systematic review. Pharmacoepidemiol Drug Saf 2012;21:141-7.

26 Noseworthy PA, Yao X, Abraham NS, et al. Direct comparison of dabigatran, rivaroxaban, and apixaban for effectiveness and safety in nonvalvular atrial fibrillation. Chest 2016;150:1302-12.

27 Yao X, Shah ND, Sangaralingham LR, et al. Non-Vitamin K antagonist oral anticoagulant dosing in patients with atrial fibrillation and renal dysfunction. J Am Coll Cardiol 2017;69:2779-90. 
28 Fan J, Arruda-Olson AM, Leibson CL, et al. Billing code algorithms to identify cases of peripheral artery disease from administrative data. J Am Med Inform Assoc 2013;20:e349-54.
29 Yao X, Tangri N, Gersh BJ, et al. Renal outcomes in anticoagulated patients with atrial fibrillation. J Am Coll Cardiol 2017;70:2621-32. 